\title{
Robótica: una nueva rama en cirugía plástica
}

\author{
Robotics: a new branch in plastic surgery \\ Dr. Luis César Valencia-García,* Dr. Patricio Canepa-Fernández,; \\ Dr. Roberto Matabuena-Tamez, ${ }^{\S}$ Dr. René Eduardo Oviedo-García
}

Palabras clave: Cirugía robótica, microcirugía, Da Vinci, cirugía plástica.

Keywords: Robotic surgery, microsurgery, Da Vinci, plastic surgery

\footnotetext{
* Cirujano Plástico. Servicio de Cirugía Plástica y Reconstructiva, Hospital General «Dr. Rubén Leñero». México.

* Residente de Cirugía Plástica y Reconstructiva, Hospital General «Dr. Rubén Leñero». México. § Cirujano Plástico, Hospital Ángeles Querétaro. México. " Residente de Cirugía Plástica y Reconstructiva, Centro Médico ISSEMyM Toluca. México.

Recibido:

09 noviembre 2020

Aceptado:

15 febrero 2021
}

\section{RESUMEN}

La cirugía reconstructiva siempre ha sido un reto quirúrgico. Los atributos de la cirugía robótica hacen que sea una opción muy atractiva para la microcirugía, una disciplina que demanda una óptima visualización, disminución del temblor, habilidad técnica y una manipulación quirúrgica precisa. Es importante actualizar a la comunidad en cirugía plástica sobre la expansión de la robótica en nuestra especialidad, demostrando cómo las características del robot pueden contribuir a mejorar la seguridad y los resultados quirúrgicos. Se realizó una revisión bibliográfica en las bases de datos de PubMed y Medline, utilizando los siguientes términos de búsqueda de forma individual o combinada: robot, robótica en cirugía plástica, microcirugía robótica. Seleccionamos 16 artículos y los principales resultados se muestran en el presente trabajo. También discutimos las controversias que existen, costos y curva de aprendizaje. El aumento en los procedimientos de mínima invasión ha hecho que éstos vayan al alza. En cirugía plástica son pocas sus aplicaciones hasta el momento, pero sin duda a futuro se verá un incremento. El costo es la desventaja principal, pero si el robot es utilizado por diferentes especialidades en un hospital, a la larga el dispositivo podría tener una relación costo/beneficio positivo. La cirugía robótica es el siguiente paso en la evolución de la cirugía de mínima invasión, ya que brinda niveles suprahumanos de precisión y una visualización en 3D en alta definición que puede expandir la capacidad de los cirujanos plásticos para lograr procedimientos complejos de una forma más segura y efectiva.

\section{ABSTRACT}

Reconstructive surgery has always been a surgical challenge. The attributes of robotic surgery make it a very attractive option for microsurgery, a discipline that demands optimal visualization, decreased tremor, technical skill, and precise surgical manipulation. It is important to update the plastic surgery community regarding the expansion of robotics in our specialty, demonstrating how the characteristics of the robot can contribute to improve safety and surgical results. We did a bibliographic review in the PubMed and Medline databases, using the following search terms individually or in combination: robot, robotics in plastic surgery, robotic microsurgery. We selected 16 articles and the main results are presented in this work. We also discuss the controversies that exist, costs and the learning curve. The increase in minimally invasive procedures has made these procedures rise. In plastic surgery there are few applications so far, but; undoubtedly, in the future, there will be an increase. Cost is the main disadvantage; but, if the robot is used by different specialties in a hospital, the device could have a positive cost/benefit ratio in the long run. Robotic surgery is the next step in the evolution of minimally invasive surgery, providing superhuman levels of precision and high definition $3 D$ visualization that can expand the ability of plastic surgeons to achieve complex procedures in a safer and more effective way.

\section{INTRODUCCIÓN}

T a cirugía reconstructiva siempre ha sido un reto quirúrgico, en el que no sólo se busca minimizar la morbilidad de la zona do- nadora, también necesita niveles superiores de precisión y meticulosidad para lograr buenos resultados. En esta nueva era en que la robótica busca simplificar y automatizar procesos, la cirugía ha sido un área de oportunidad muy

Citar como: Valencia-García LC, Canepa-Fernández P, Matabuena-Tamez R, Oviedo-García RE. Robótica: una nueva rama en cirugía plástica. Cir Plast. 2021; 31 (1): 43-49. https://dx.doi.org/10.35366/101086 
importante, ya que proporciona ventajas únicas para enfrentar muchas limitantes de las técnicas tradicionales. Esto incluye un acceso con mínima invasión, una imagen con mayor resolución y claridad, gran precisión quirúrgica que filtra el temblor, siete grados de libertad con movimientos similares a la muñeca y una posición ergonómica durante el procedimiento. ${ }^{1}$ Es por ello que muchas especialidades quirúrgicas ya están aplicando esta tecnología. ${ }^{2}$

En cirugía plástica no estamos muy relacionados con procedimientos de mínima invasión, ésta es la razón que la inquietud por esta tecnología ha llegado más tarde a nuestra especialidad y en la actualidad son pocos los autores que se encuentran involucrados en el tema. Hasta el momento son escasas las aplicaciones en cirugía plástica; sin embargo, los atributos de la cirugía robótica hacen que sea una opción muy atractiva, principalmente para la microcirugía, una disciplina que demanda una óptima visualización, disminución del temblor, habilidad técnica y una manipulación quirúrgica precisa. ${ }^{2,3}$

El objetivo de este trabajo es actualizar a la comunidad en cirugía plástica sobre la expansión de la robótica en nuestra especialidad, demostrando cómo las características del robot pueden contribuir a mejorar la seguridad y los

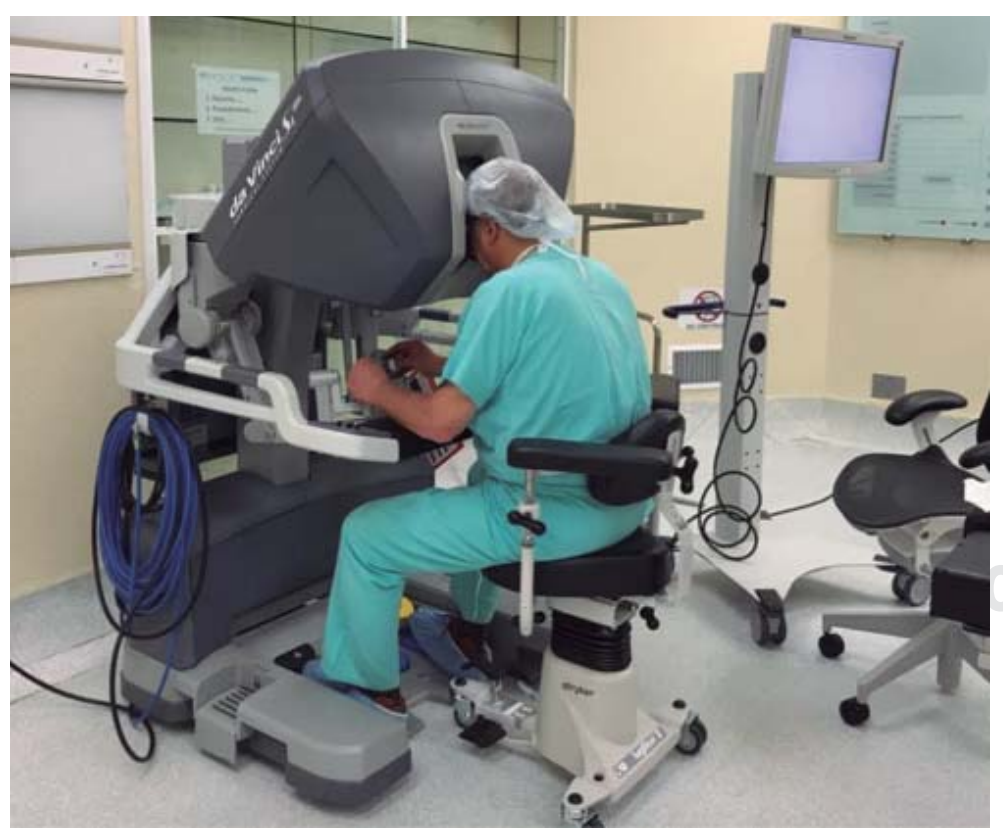

Figura 1: Robot Da Vinci. resultados quirúrgicos. También discutimos las controversias que existen en su aplicabilidad, costos y curva de aprendizaje.

\section{MATERIAL Y MÉTODO}

Se realizó una revisión bibliográfica en las bases de datos de PubMed y Medline, se utilizaron los siguientes términos de búsqueda de forma individual o combinada: robot, robótica en cirugía plástica, microcirugía robótica. Se analizaron los resúmenes para su inclusión y artículos con referencias cruzadas. Se incluyeron todos los tipos de estudio y se excluyeron aquellos no relacionados con cirugía plástica.

La búsqueda arrojó un total de 14,652 ítems bajo el término «robot» y 1,566 artículos de revisión. Bajo el término «robótica en cirugía plástica» 156 ítems y 11 artículos de revisión. Con el término «robótica en microcirugía» 464 ítems y 106 artículos de revisión. Duplicaciones y artículos no publicados en inglés fueron eliminados. Los artículos que investigan específicamente el uso de la tecnología robótica activa en un contexto reconstructivo y de cirugía plástica fueron finalmente elegidos para revisión, método que permitió seleccionar 16 artículos para este trabajo.

\section{HISTORIA}

La cirugía robótica fue un proyecto iniciado por la NASA (National Aeronautics and Space Administration) y la milicia de los Estados Unidos de América, con el propósito de desarrollar un sistema telerrobótico para realizar procedimientos quirúrgicos a distancia, debido a su potencial aplicación en guerras y en viajes al espacio. La tecnología fue adquirida por Intuitive Surgical (Sunnyvale, CA), creadores del sistema robótico más utilizado en la actualidad, el Da Vinci (Figura 1). ${ }^{1-4}$

Las aplicaciones de la cirugía robótica son amplias en especialidades como urología, cirugía general, ginecología y otorrinolaringología, a diferencia de cirugía plástica donde han sido mucho menores. ${ }^{3}$

Desde 2003, investigadores - como el doctor Amir Ibrahim - se han dado a la tarea de ahondar su aplicación en cirugía plástica. ${ }^{1}$ De los primeros cirujanos en llevar a cabo un pro- 


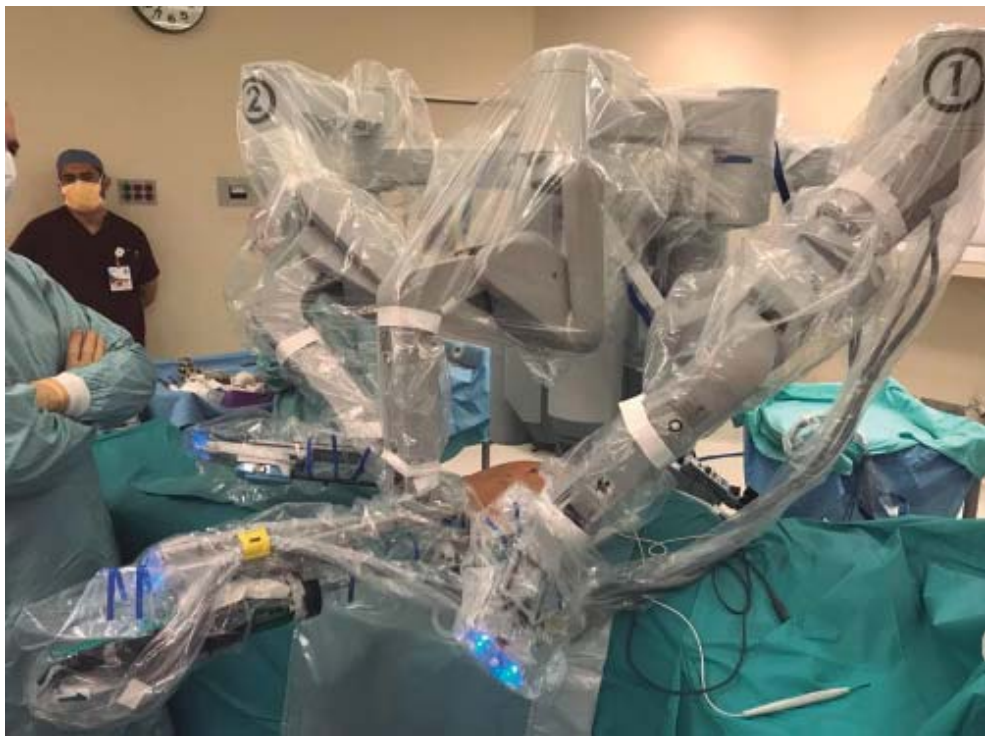

Figura 2: Cuatro brazos del robot armados.

cedimiento de tal riesgo fueron Katz y colaboradores, en el Johns Hopkins University School of Medicine a principios de 2005, quienes realizaron de manera exitosa un colgajo libre experimental en un modelo porcino utilizando un robot Da Vinci. La disección y toma del colgajo se hicieron de la manera convencional, el robot se usó para completar la adventectomía y las anastomosis microvasculares. ${ }^{5}$

El primer reporte de seis anastomosis microvasculares en ratas mostró un tiempo significativamente menor contra las anastomosis convencionales, sin mostrar diferencia en cuanto a fallas técnicas. ${ }^{6}$ En 2010 se introdujo la disección con robot del músculo dorsal ancho posterior a su investigación en modelos de cadáver. ${ }^{7}$

\section{DA VINCI}

El robot Da Vinci opera en una configuración maestro-esclavo; el sistema no actúa de manera autónoma sin la intervención directa del cirujano y no funciona con secuencias automáticas. El dispositivo quirúrgico de cuatro brazos permite adaptar instrumentos intercambiables en tres de sus brazos, dejando uno para el endoscopio (Figuras 2 y 3). Cámaras compensadoras de doble alineación permiten alimentar a los visores en la consola, lo cual proporciona al cirujano una imagen tridimensional de alta resolución del campo quirúrgico. Los movimientos de la mano del cirujano en la consola de mando se traducen en movimientos de los instrumentos. Los puntos de articulación de los instrumentos permiten siete grados de libertad, similar al brazo humano; en cambio, la laparoscopia estándar proporciona sólo cuatro. Además, el temblor de la mano se elimina con la escala de movimiento. Los movimientos son replicados en tiempo real y pueden ser disminuidos a escala para una mayor precisión. La cabeza de los instrumentos gira $360^{\circ}$ y los brazos del robot pueden mantener posiciones estáticas. El cirujano se sienta en la consola separada de la mesa de operaciones, por lo general en la misma habitación (Figura 4).,2

\section{APLICACIONES CLÍNICAS EN CIRUGíA PLÁSTICA}

Con la eliminación del temblor, escala de movimiento 5:1, óptica 3D en alta definición y magnificación hasta $10 \times$, el robot logra un nivel de precisión suprahumano y una configuración ideal para realizar la manipulación delicada que se requiere. Asimismo, permite realizar accesos con incisiones mínimas, lo que mejora el resultado funcional. ${ }^{9}$

Hasta este momento se han establecido tres aplicaciones solidas en esta área:

1. Cirugía robótica transoral para reconstrucción de cabeza y cuello.

La cirugía robótica transoral (TORS) — que de manera segura puede extirpar tumores de cabeza y cuello sin necesidad de realizar mandibulectomías y la disección cervical asistida por robot usando un acceso retroauricular que hacen la cicatriz de la incisión menos perceptible- son consideradas cirugías mínimamente invasivas; no obstante, estas incisiones son problemáticas para la cirugía plástica, porque el espacio disponible para realizar la cirugía reconstructiva puede resultar restringido. Para optimizar la función fisiológica de la orofaringe y minimizar las deficiencias funcionales es necesario preservar un esfínter velofaríngeo competente, un sello hermético entre la faringe y el cuello, una sensación y volumen adecuados en la base de la lengua. ${ }^{10}$ 
La reconstrucción robótica es una solución a estos problemas. La precisión del robot y la visualización mejorada en espacios confinados lo hacen más apropiado para la disección del cuello y la reconstrucción con TORS. En otras palabras, se necesita la inserción de un colgajo libre asistido por robot y la microanastomosis para reconstruir el defecto orofaríngeo resultante de TORS en un espacio confinado, aprovechando la imagen magnificada de alta resolución y la precisión de los instrumentos. ${ }^{10}$ El robot permite que con estas ventajas se pueda realizar la anastomosis sin necesidad de accesos extensos que incrementen la morbilidad.

\section{Microcirugía.}

La microcirugía constituye una de las herramientas más poderosas en el armamento del cirujano reconstructivo. A pesar de que las técnicas actuales en microcirugía son efectivas y confiables, presentan limitantes como una curva prolongada de aprendizaje, la inhabilidad para eliminar el temblor y contar con instrumentos rígidos no articulados. El robot con una visión 3D, con instrumentos completamente articulados, movimientos a escala, filtración del temblor y operación en una posición ergonómica, minimiza la fatiga del cirujano y logra una mayor precisión.

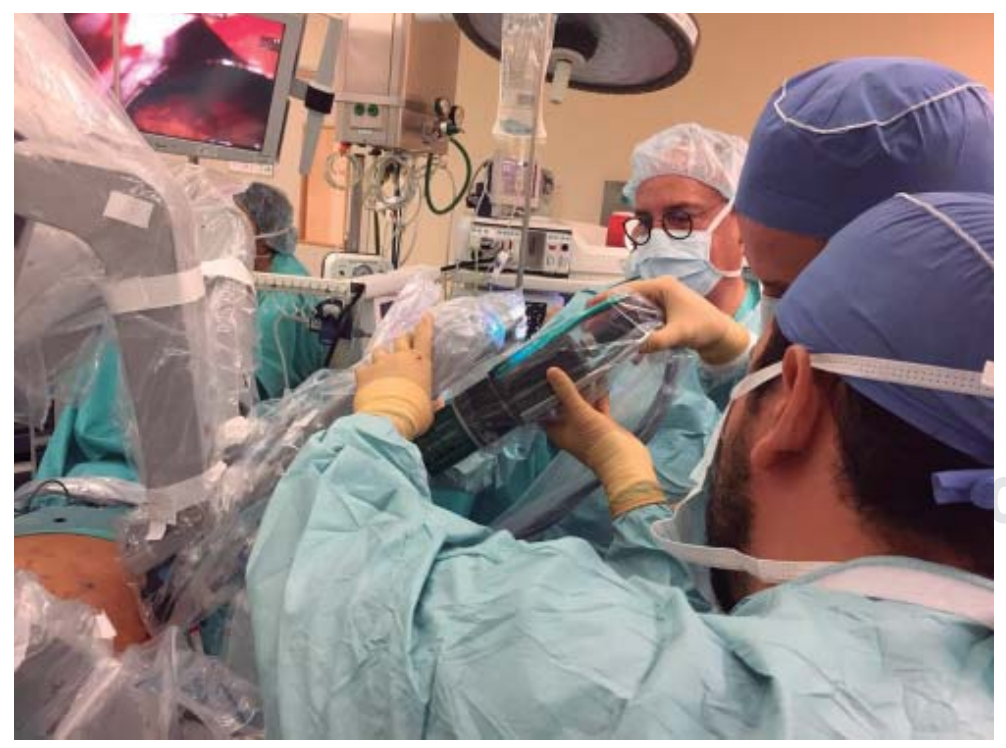

Figura 3: Dispositivo con brazo para endoscopio.

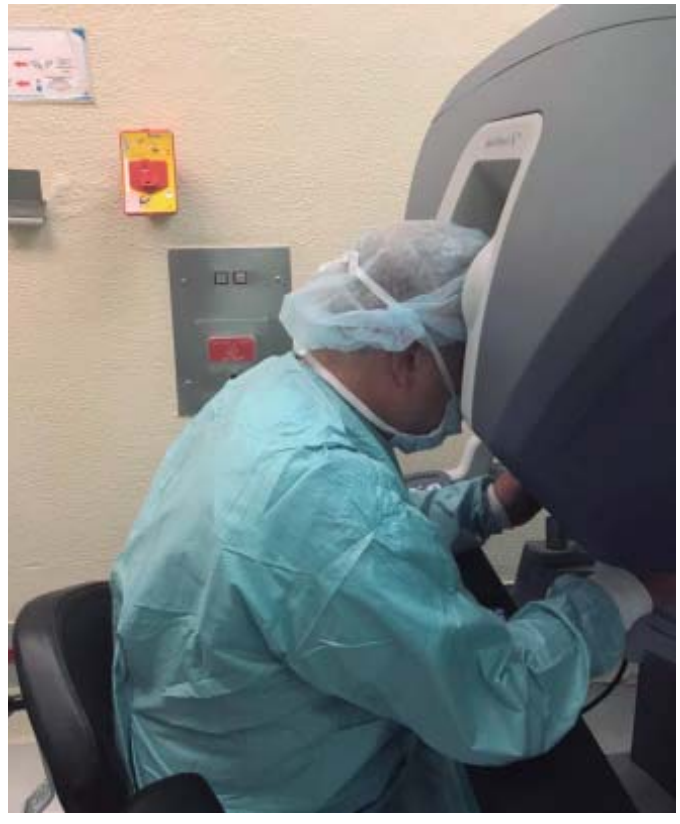

Figura 4: Movimientos del cirujano replicados en tiempo real.

Estas ventajas han hecho que la microcirugía asistida con robot se aplique en anastomosis de pequeños vasos, reparaciones microneurales y en supermicrocirugía para procedimientos como el bypass linfático-venoso términoterminal.

Hay estudios que reflejan una rápida curva de aprendizaje y una disminución de tiempo quirúrgico en microcirugía robótica, similares a los resultados que se muestran en otras especialidades. En el caso de microanastomosis, diversos autores señalan que el hecho de no tener percepción táctil no dificulta realizar las anastomosis cuando se cuenta con experiencia suficiente, pero pudiera ser algo problemático en los cirujanos con poca experiencia.

Otra de sus ventajas es que se puede maniobrar en lugares pequeños y anatómicamente confinados como axila o extremidades.

Aún no existen instrumentos especializados para microcirugía, seguramente en cuanto se expanda esta aplicación se desarrollarán y contribuirán a disminuir los tiempos. Esta tecnología también hará que los beneficios de la mano humana se optimicen con la precisión del robot para dar resultados más seguros y predecibles. ${ }^{1,2,7,9,11}$ 
3. Disección de colgajos musculares.

Los colgajos del músculo recto abdominal y dorsal ancho han sido los caballos de batalla en la cirugía reconstructiva desde finales de 1970. Su disección requiere incisiones largas que van desde los 15 a $45 \mathrm{~cm}$ de longitud. ${ }^{12}$ Estas incisiones están asociadas con morbilidad (seroma, hernia, dolor intenso) y se localizan en abdomen o espalda con un pobre resultado estético.

En 2011 se realizó un protocolo de disección de colgajo dorsal ancho en ocho pacientes de forma exitosa y desde entonces se han realizado más de 40 casos en un solo centro hospitalario. Esta técnica novedosa se asoció con una reducción de la morbilidad en la zona donadora, con un mejor resultado estético y una estancia intrahospitalaria más corta. Esta técnica consiste en una incisión pequeña en la axila de 5 a $8 \mathrm{~cm}$ (se puede utilizar la incisión del ganglio centinela si fuera el caso) para realizar la disección del pedículo principal y dos incisiones más para los puertos (12 y $8 \mathrm{~mm}$ ) e insuflación (10 mmHg). Técnicamente ofrece ventajas superiores a la cirugía endoscópica y a la técnica abierta, y puede ser utilizado como un colgajo libre o pediculado. Sus principales indicaciones incluyen reconstrucción mamaria, deformidades en tórax y escalpes. ${ }^{12}$

En la disección del músculo recto abdominal se han notado múltiples ventajas como incisiones pequeñas, lo que reduce las complicaciones de las heridas, mejora la calidad de las cicatrices y el resultado estético. Además, preserva la fascia anterior del músculo recto, lo que disminuye la incidencia de abultamientos y hernias. Se ha utilizado principalmente para cubrir defectos en extremidades y en la región pélvica.

Una de las aplicaciones prometedoras es la exploración temprana en lesiones cerradas del plexo braquial, con la finalidad de realizar una reparación temprana, ya que permite llevar a cabo una exploración por medio de incisiones pequeñas disminuyendo las complicaciones por las disecciones extensas, la degeneración de la placa neural y la atrofia muscular; esto cambiaría los paradigmas del manejo expectante.

\section{VENTAJAS DE LA CIRUGÍA ROBÓTICA}

En muchas situaciones la cirugía robótica ofrece beneficios sobre la cirugía convencional, que incluyen menor invasión, reducción del trauma tisular, menor pérdida sanguínea, menor dolor postoperatorio y estancia hospitalaria, recuperación rápida y un pronto regreso a las actividades laborales. ${ }^{13}$

Recientemente, se publicó un artículo que demuestra una estancia hospitalaria significativamente menor entre pacientes que fueron sometidos a cirugía transrobótica oral con reconstrucción mediante colgajo radial libre contra pacientes tratados mediante labio mandibulectomía y reconstrucción con colgajo radial libre. ${ }^{14}$

Un microcirujano experimentado opera con una precisión de 50 micras, la asistencia mediante robot refina esta precisión a 5-10 micras. A nivel microscópico, los vasos sanguíneos suturados mediante asistencia con robot muestran menor daño en la pared, debido a la eliminación del temblor de la mano. ${ }^{15}$

La eliminación completa del temblor, aunado a la escala de movimientos 5:1, ofrece a los microcirujanos niveles suprahumanos de precisión. Existe un estudio que cuantificó el temblor con un sistema 3D, el cual demuestra que apoyar las muñecas disminuye el temblor de forma significativa, pero sin alcanzar la filtración generada por el robot.

\section{DESVENTAJAS DE LA CIRUGÍA ROBÓTICA}

El costo es el aspecto más debatido de la cirugía robótica y pudiera ser un obstáculo para expandir su uso en la cirugía plástica. El costo aproximado del sistema Da Vinci es de \$2.2 millones de dólares y el mantenimiento anual es de $\$ 138,000$. Muchos centros hospitalarios han aceptado el costo por diversas razones, como anticipar un incremento en el volumen de pacientes, lograr aumentar su reputación y por los beneficios académicos que esto conlleva.

El margen de utilidad por caso es la determinante final del beneficio financiero, se obtiene al restar el costo del caso por el ingreso. Para la mayoría de las cirugías el ingreso de una cirugía robótica ha resultado ser igual 
que si se realiza de forma abierta. Si bien es cierto, el costo de quirófano es mayor debido al equipo, personal y tiempo quirúrgico, pero la cirugía robótica de mínima invasión está asociada con una estancia intrahospitalaria más corta y a menos complicaciones. El hecho de que en un hospital el robot sea utilizado por múltiples especialidades permite aprovechar el dispositivo y beneficiarse de sus ventajas. Tomando en cuenta lo anterior, el costo final de un caso con cirugía robótica pudiera compararse con el de los accesos tradicionales. Para poder determinarlo se necesitará realizar estudios prospectivos a largo plazo aplicados a procedimientos de cirugía plástica que valoren el costo beneficio de esta nueva tecnología, en términos de tiempo quirúrgico, recuperación, estancia intrahospitalaria y morbilidades. ${ }^{15}$

Irónicamente el inicio de la cirugía laparoscópica estuvo asociado con una larga curva de aprendizaje y un costo excesivo para los sistemas de salud. La menor curva de aprendizaje demostrada en cirugía robótica pudiera significar menor costo en su implementación, así como introducirlo en diferentes especialidades. Hasta el momento no existe un estudio que examine el potencial económico.

Otra de las limitantes es el entrenamiento. Como prerrequisito se necesita un entendimiento en el aspecto mecánico, dinámico y cinético de la robótica; por lo tanto, los cirujanos necesitan dedicar tiempo y energía para entender los matices de la mecánica robótica para ser capaces de solucionar problemas cuando la máquina no funcione de manera óptima. A pesar de los avances tecnológicos, hasta el momento no se ha ideado un curso de aprendizaje específico por módulos y con certificación para cirugía plástica, lo que hace que el método de aprendizaje sea a base de prueba y error, haciéndolo completamente dependiente del número de casos ejecutados.

Por lo anterior, un simulador podría ser una alternativa para adquirir las habilidades quirúrgicas robóticas, familiarizar al cirujano con la manipulación de los instrumentos en un sistema tridimensional y desarrollar una retroalimentación de la fuerza necesaria para manipular diferentes tejidos. De esta forma, los cirujanos podrán llevar registro de su progreso y ser supervisados por un mentor, inclusive a distancia. Esto permite adquirir las habilidades necesarias de forma más rápida y sin comprometer la seguridad del paciente. ${ }^{15}$

Existen limitaciones técnicas de los modelos robóticos actuales aplicados a la microcirugía, ya que no cuentan con instrumentos finos específicos para microcirugía, presentan una óptica inferior comparada con la de un microscopio y la falta de la percepción táctil de los tejidos; sin embargo, con la expansión de la cirugía robótica en estos días, no tardará mucho que los fabricantes tomen en cuenta estos aspectos. ${ }^{1,3,15}$

\section{CIRUGÍA ESTÉTICA}

Es importante mencionar que la cirugía estética no se ha prestado a la aplicación de la tecnología robótica y tal vez nunca lo haga. Aún no se cuenta con algún reporte de cirugía robótica en un procedimiento estético; hasta el momento el único robot involucrado con algún procedimiento estético es para injerto capilar.

La clave del éxito en cirugía estética está en un análisis preoperatorio adecuado, marcaje, valoración táctil repetida de los tejidos y la tensión en las suturas. La mayor parte de esto es logrado por las manos de los cirujanos, las cuales son capaces de identificar deformidades e irregularidades en el contorno. El sentido del tacto también es crítico para minimizar lesiones en los tejidos, y al trabajar con un robot el cirujano depende principalmente de las señales visuales y no de la retroalimentación táctil. Se han desarrollado sistemas con sensores que dan retroalimentación de la fuerza, pero no han funcionado del todo por el tamaño, diseño, costo, compatibilidad y la capacidad de tolerar los procesos de esterilización. Por lo tanto, es difícil que se pueda reemplazar el sentido del tacto que es crítico en la cirugía estética, como tampoco las habilidades y el toque artístico del cirujano, que son elementos que logran un adecuado resultado estético más allá de una precisión técnica y una ejecución mecánica programada.

\section{DISCUSIÓN}

La mayor demanda por parte de los pacientes en los procedimientos de mínima invasión, y 
los avances tanto en sistemas computacionales como en ingeniería robótica, han hecho que estos procedimientos vayan al alza. En cirugía plástica son pocas sus aplicaciones hasta el momento, pero sin duda veremos un incremento en los próximos años. Se va a requerir de estudios prospectivos que analicen el costo-beneficio a largo plazo, en los que se consideren factores como tiempo quirúrgico, estancia intrahospitalaria, complicaciones y resultados, entre otros, para poder determinar si realmente es viable aplicar una tecnología en la que el costo es la desventaja principal. Sin embargo, se piensa que si el robot es utilizado por diferentes especialidades en un hospital, el dispositivo a la larga podría tener una relación costo-beneficio positiva.

La medicina clínica ha adoptado con más lentitud esta práctica por temas de seguridad, costos y factibilidad. ${ }^{3}$ Pero lo que es un hecho es que los nuevos robots han cambiado su papel en cirugía como ayudantes a ser las manos del primer cirujano a través de una interfase por computadora. $2,7,16$

\section{CONCLUSIONES}

La cirugía robótica es el siguiente paso en la evolución de la cirugía de mínima invasión, la cual brinda niveles suprahumanos de precisión y una visualización en 3D incomparable que puede expandir la capacidad de los cirujanos plásticos para lograr procedimientos complejos de una forma más segura y efectiva.

En cirugía plástica, esta técnica se encuentra en una etapa muy temprana; sin embargo, el futuro que le espera es favorable, con el desarrollo de plataformas tecnológicas, mejoría en los sistemas de aprendizaje, adaptación del instrumental quirúrgico y la disminución de los costos (la cual eventualmente afecta a todas las revoluciones tecnológicas). Se espera que la robótica en cirugía plástica florezca y se expanda en los próximos años. Debemos abrir nuestro panorama ante esta revolución tecnológica que ha traído consigo avances significativos, como la impresión 3D, la robótica y aplicaciones de monitoreo vía smartphone, entre otras. Por lo tanto, invitamos a los lectores a aceptar las ventajas que nos ofrece la tecnología y a descubrir nuevas aplicaciones que nos permitan ofrecer un mejor manejo a los pacientes de forma segura, basados en evidencia clínica.

\section{REFERENCIAS}

1. Ibrahim A, Sarhane K, Selber J. Robotics in plastic surgery. Curr Surg Rep 2016; 4 (9): 1-9.

2. Katz R, Taylor J, Rosson G, Brown P, Singh N. Robotics in plastic and reconstructive surgery: use of a telemanipulator slave robot to perform microvascular anastomoses. J Reconstr Microsurg 2006; 22 (1): 53-58.

3. Saleh DB, Syed M, Kulendren D, Ramakrishnan $\mathrm{V}$, Liverneaux P. Plastic and reconstructive robotic microsurgery - a review of current practices. Ann Chir Plast Esthet 2015; 60 (4): 305-312.

4. Selber J. Robotics in plastic surgery. Semin Plast Surg 2014; 28: 3-4.

5. Ibrahim A, Sarhane K, Baroud J, Atiyeh B. Robotics in plastic surgery, a review. Eur J Plast Surg 2012; 35: 571-578.

6. Li RA, Jensen J, Bowersox JC. Microvascular anastomoses performed in rats using a microsurgical telemanipulator. Comput Aided Surg 2000; 5 (5): 326332.

7. Nehme J, Neville J, Nehme A. The use of robotics in plastic and reconstructive surgery: a systematic review. JPRS Open 2017; 13: 1-10.

8. Hassanein A, Mailey B, Dobke M. Robot assisted plastic surgery. Clin Plast Surg 2012; 39: 419-424.

9. Ibrahim A, Sarhane K, Selber J. New frontiers in roboticassisted microsurgical reconstruction. Clin Plast Surg 2017; 44 (2): 415-423.

10. Song HG, Yun IS, Lee WJ, Lew DH, Rah DK. Robotassisted free flap in head and neck reconstruction. Arch Plast Surg 2013; 40: 353-358.

11. Saraf $f$. Role of robot assisted microsurgery in plastic surgery. Indian J Plast 2006; 39 (1): 57-61.

12. Selber J, Baumann D, Holsinger C. Robotic latissimus dorsi muscle harvest: a case series. Plast Reconstr Surg 2012; 129 (6): 1305-1312.

13. Ponnusamy K, Mohr C, Curet MJ. Clinical outcomes with robotic surgery. Curr Probl Surg 2011; 48 (9): 577-656.

14. Biron VL, O'Connell DA, Barber B et al. Transoral robotic surgery with radial forearm free flap reconstruction: case control analysis. J Otolaryngol Head Neck Surg 2017; 46 (1): 20.

15. Selber JC. Robotic latissimus dorsi muscle harvest. Plast Reconstr Surg 2011; 128 (2): 88-90.

16. Selber J. Can I make robotic surgery make sense in my practice? Plast Reconstr Surg 2017; 139 (3): 781-792.

Conflicto de intereses: Los autores declaran no tener conflicto de intereses.

Correspondencia:

Dr. Luis César Valencia-García

Servicio de Cirugía Plástica, Estética y Reconstructiva, Hospital General «Dr. Rubén Leñero».

Salvador Díaz Mirón s/n,

Col. Santo Tomás, 11340,

Alcaldía Miguel Hidalgo,

Ciudad de México, México.

E-mail: luiscesarvalencia@gmail.com 